\title{
Retrospective case studies of the efficacy of caprylic triglyceride in mild-to-moderate Alzheimer's disease
}

This article was published in the following Dove Press journal:

Neuropsychiatric Disease and Treatment

22 October 2013

Number of times this article has been viewed

\author{
Steven Douglas Maynard ${ }^{1,2}$ \\ Jeff Gelblum ${ }^{3}$ \\ 'Union Associated Physicians Clinic, \\ ${ }^{2}$ Indiana University School of \\ Medicine, Terre Haute, IN, ${ }^{3}$ Mt Sinai \\ Medical Center of Miami, Aventura \\ Hospital, Aventura, FL, USA
}

\begin{abstract}
Under normal conditions, the adult human brain is fueled primarily by glucose. A prominent feature of Alzheimer's disease (AD) is region-specific decreases in cerebral glucose metabolism. Ketone bodies are a group of compounds produced from fat stores during periods of low glucose availability that can provide an alternative to glucose for brain metabolism. Consumption of sufficient quantities of caprylic triglyceride (CT) increases plasma concentrations of ketone bodies and may be beneficial in conditions of compromised glucose metabolism, such as AD. The present study describes the use of $\mathrm{CT}$ in mild-to-moderate $\mathrm{AD}$ in routine clinical practice. Case records from eight patients with extensive monitoring of cognitive function using the Mini-Mental State Examination (MMSE) and who had received CT for $\geq 6$ months were reviewed. All were outpatients aged $\geq 50$ years, cared for in standard practice, had a diagnosis of probable AD of mild-to-moderate severity (MMSE 14-24), and had received CT for at least 6 months in addition to other approved pharmacotherapy for AD. Response to CT administration as measured by MMSE scores varied by patient. However, the rate of decline in MMSE scores appeared slower than previously published reports for patients treated with pharmacotherapy alone. Profiling of individual patients may provide insight regarding those most likely to benefit from addition of CT to currently approved AD pharmacotherapy.
\end{abstract}

Keywords: ketosis, cognition, Alzheimer's disease, metabolism, glucose

\section{Introduction}

Alzheimer's disease (AD) is a progressive neurologic disorder that primarily afflicts the elderly, and is the most common form of dementia in patients over the age of 65 years. In the US, an estimated 5.4 million people had AD in 2012. This includes 5.2 million people $\geq 65$ years of age with late-onset AD and about 200,000 people aged $<65$ years with early-onset AD. ${ }^{1}$ Current drug treatments for AD are limited to cholinesterase inhibitors (donepezil, galantamine, rivastigmine, and tacrine) and the $\mathrm{N}$-methyl-D-aspartate receptor antagonist, memantine. While effective, these treatments are considered symptomatic and disease progression continues. ${ }^{2}$ Development and evaluation of new AD therapies is urgently needed.

Medical foods are a growing category of options for AD patients. Medical foods are defined in section 5(b) of the Orphan Drug Act (21 U.S.C. 360ee) as "a food which is formulated to be consumed or administered enterally under the supervision of a physician and which is intended for the specific dietary management of a disease or condition for which distinctive nutritional requirements, based on recognized scientific principles, are established by medical evaluation". ${ }^{3}$ Medical foods are regulated, but not approved, by the US Food and Drug Administration. Types of medical foods used 
in AD include compositions of omega-3 fatty acids, uridine, and choline; ${ }^{4}$ a combination of folate, vitamin $\mathrm{B}_{6}$, alphatocopherol, S-adenosyl methionine, $\mathrm{N}$-acetyl cysteine, and acetyl-L-carnitine, ${ }^{5}$ and ketogenic agents, such as caprylic triglyceride (CT). ${ }^{6}$

Caprylic triglyceride-based medical foods have been prescribed to patients with mild-to-moderate $\mathrm{AD}$ for several years based on results in clinical trials. ${ }^{6,7}$ The rationale for induction of ketosis in mild-to-moderate AD centers on the well-recognized regional decreased cerebral glucose metabolism that characterizes AD. ${ }^{8}$ Under most normal conditions, the adult human brain relies almost exclusively on glucose for energy generation. However, the brain can also utilize compounds called ketone bodies as an energy source, and will do so in conditions that include neonatal development and starvation. ${ }^{9}$ Ketone bodies are an efficient fuel for cells that can supplement glucose metabolism in the brain when they are present in the circulation. ${ }^{10}$ Caprylic triglyceride is classified as a medium chain triglyceride and is known to induce ketosis without the need to change diet. ${ }^{11}$ Results from a recent chart review/caregiver survey indicated efficacy of addition of CT to pharmacotherapy for stabilizing clinical status as assessed by the treating physicians. ${ }^{12}$ A small number of patients whose records were included in this review had repeated documentation of cognitive function with the Mini-Mental State Examination (MMSE) both before and after addition of $\mathrm{CT}$ to the therapeutic regimen. Results from these cases permit a more complete description of the effects of adding CT to pharmacotherapy in patients with AD. This paper summarizes results from these eight patients.

\section{Case series}

The cases described are from a larger sample of male and female outpatients aged $\geq 50$ years with a diagnosis of probable AD of mild-to-moderate severity at initiation of CT therapy (MMSE 14-24 inclusive, if available) and receipt of CT for $\geq 6$ months. Patients were excluded if diagnosed with a comorbid disease known to adversely impact cognitive function (eg, Parkinson's disease, history of cerebral infarcts, dementia with Lewy bodies, vascular dementia). In addition, none of the eight patients described in this report had evidence of pancreatic deficiency, diabetes, or alcohol abuse in their medical records.

This study was conducted under institutional review board approval and is registered at http://www.clinicaltrials.gov/ct2/ show/NCT01538212. Patients or their caregivers provided informed written consent for extraction of all information presented in this paper from their medical records. All of the patients $(n=55)$ whose records were included in the study had assessments that included Physician's Overall Assessment of Patient Status, MMSE (if available), change in patient's living situation, medication changes for the treatment of AD, caregivers' assessment of changes in the patient's memory, ability to carry out instrumental activities of daily living, and adverse events. This case review includes all patients $(n=8)$ who had at least two MMSE evaluations both before initiation of CT and at least three measurements after initiation of this therapy. The demographic and clinical characteristics of the eight cases reported in detail here (Table 1) are consistent with those from the larger sample of patients included in the overall record review. ${ }^{12}$ Results for all MMSE evaluations for each patient are shown in Figure 1 and mean MMSE scores (first recorded and first and last on $\mathrm{CT}$ ) are shown in Figure 2.

\section{Case I}

In the first case, the patient's MMSE score was 25 at the time of diagnosis. He was treated with donepezil $10 \mathrm{mg}$ once daily and memantine $10 \mathrm{mg}$ twice daily. The patient was also receiving quetiapine beginning in January 2011 for treatment of agitation. Over the period from April 2007 until September 2009, the patient's MMSE score declined from 25 to 15, an average annual change of -3.4 points (Figure 1A). In September 2009, CT (20 g/day, the recommended dose) was added to therapy due to the decline in the patient's cognitive function.

Table I Patient characteristics

\begin{tabular}{|c|c|c|c|c|c|c|c|}
\hline Patient & Gender & $\begin{array}{l}\text { Age, } \\
\text { years }\end{array}$ & Race & $\begin{array}{l}\text { Marital } \\
\text { status }\end{array}$ & $\begin{array}{l}\text { Age at diagnosis, } \\
\text { years }\end{array}$ & $\begin{array}{l}\text { Basis of } \\
\text { diagnosis }\end{array}$ & $\begin{array}{l}\text { Living } \\
\text { arrangement }\end{array}$ \\
\hline I & M & 94 & White & Married & 89 & SS, MRI, CT & Assisted living facility \\
\hline 2 & M & 91 & White & Married & 89 & SS, MRI, CT & With caregiver \\
\hline 3 & $\mathrm{~F}$ & 78 & White & Married & 74 & $\mathrm{SS}, \mathrm{CT}$ & With caregiver \\
\hline 4 & M & 84 & African-American & Married & 82 & SS, EEG, CT & With caregiver \\
\hline 5 & $\mathrm{~F}$ & 81 & White & - & 78 & $\mathrm{SS}, \mathrm{CT}$ & With caregiver \\
\hline 6 & $M$ & 86 & White & Married & 83 & SS, MRI, CT & With caregiver \\
\hline 7 & $M$ & 74 & White & Married & 70 & SS, EEG, MRI, CT & With caregiver \\
\hline 8 & $M$ & 88 & African-American & Married & 85 & SS, CT & With caregiver \\
\hline
\end{tabular}

Abbreviations: CT, cognitive testing; EEG, electroencephalogram; F, female; M, male; MRI, magnetic resonance imaging; SS, signs and symptoms. 

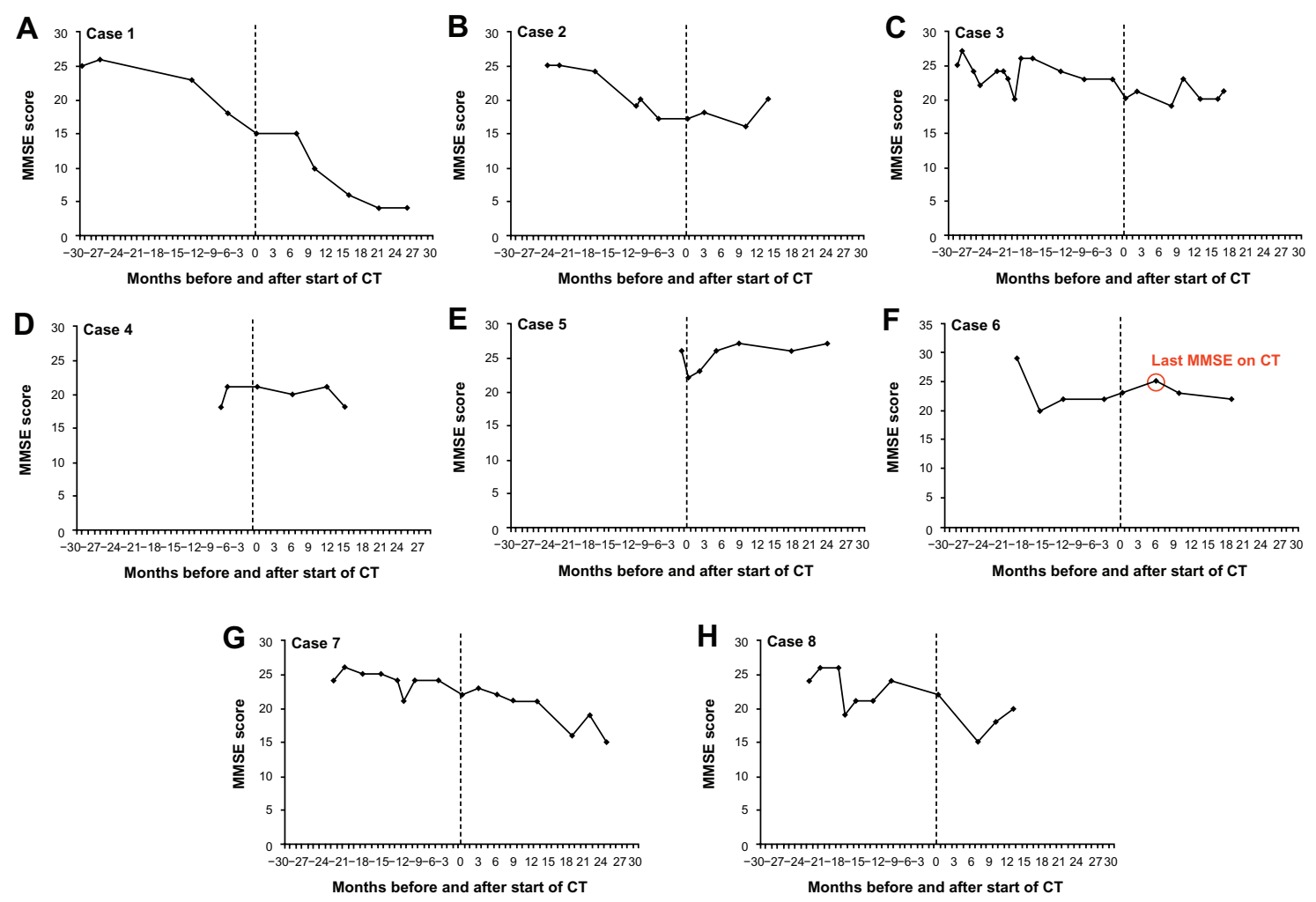

Figure I (A-H) Changes in MMSE scores for the cases reviewed (the dashed line in each panel indicates initiation of CT).

Abbreviations: CT, caprylic triglyceride; MMSE, Mini-Mental State Examination.

The patient remained on $\mathrm{CT}$ and the MMSE continued to decline, reaching a score of 6 by January 2011, an average decline of -6.7 points/year. The primary physician noted that the patient progressed from mild $\mathrm{AD}$ to severe $\mathrm{AD}$ over the course of CT and his change in status was described as moderate worsening. No adverse events related to CT were recorded and no caregiver assessment was available. It should be noted that CT was initiated in this patient when he had moderate $\mathrm{AD}$ and was continued as he progressed to severe dementia.

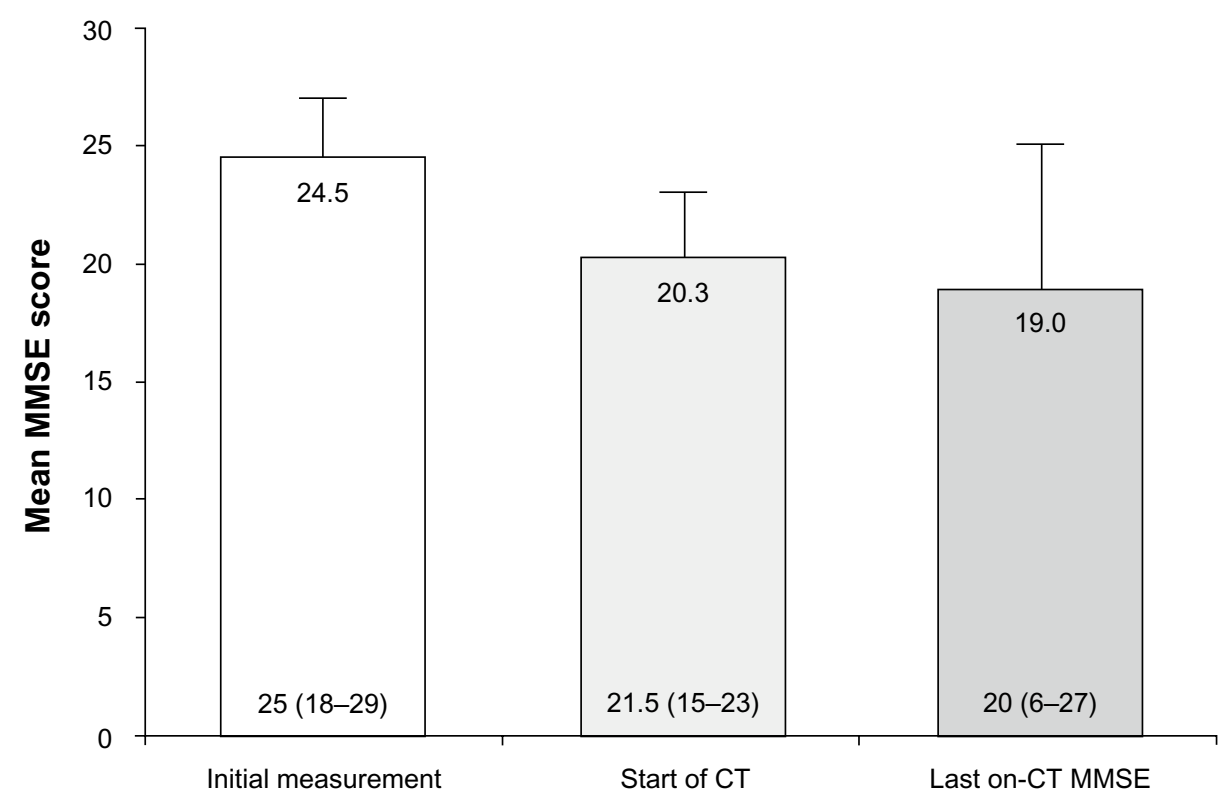

Figure 2 Mean values for MMSE at initial measurement, start of CT, and last measurement on CT (error bars are standard deviations, with median and range shown in each bar). Abbreviations: CT, caprylic triglyceride; MMSE, Mini-Mental State Examination. 


\section{Case 2}

The second patient had an MMSE score of 25 at the time of diagnosis (Figure 1B). He was initially treated with donepezil $10 \mathrm{mg}$ once daily beginning in March 2009, but this treatment was terminated in November 2009 due to drowsiness. Galantamine $16 \mathrm{mg}$ once daily was initiated in November 2009 and memantine $10 \mathrm{mg}$ twice daily was added to therapy in June 2010. Both of these treatments continued. The patient was not receiving any other psychotropic medications. Caprylic triglyceride $20 \mathrm{~g}$ /day was initiated in March 2011 and the patient's MMSE score at this evaluation was 17 (average annual change of -4.0 points). Follow-up MMSE scores were available through May 2012; at this time, the score was 20 (average annual change of +2.6 points). The patient reported no adverse events over the 14 months of CT, and the physician described his condition as stable. Caregiver evaluation was not available for this patient.

\section{Case 3}

The third patient had an MMSE score of 25 that declined to 20 by January 2011 (annual change of -2.5 points, Figure 1C). At the initiation of CT therapy ( $20 \mathrm{~g} /$ day) in January 2011, she was using the $9.5 \mathrm{mg}$ rivastigmine patch and presented with an MMSE of 20. She was also being treated with escitalopram, alprazolam, quetiapine, and aripiprazole. The patient continued on CT and rivastigmine, and the last follow-up MMSE score in July 2012 was 21 (annual change of +0.7 points from initiation of CT, Figure 1C). The treating physician reported a diagnosis of mild $\mathrm{AD}$ at the beginning of $\mathrm{CT}$ and moderate $\mathrm{AD}$ at the last available evaluation. The physician rated the patient's condition over the 19 months as stable. No adverse events related to CT were reported. The caregiver evaluation reported some deterioration.

\section{Case 4}

The fourth case presented with an MMSE score of 18 in August 2010. Relevant AD medications included galantamine $8 \mathrm{mg}$ once daily, followed by galantamine extendedrelease $16 \mathrm{mg}$ once daily and memantine $10 \mathrm{mg}$ twice daily. The patient was not taking any other psychotropic medications. By February 2011, the MMSE score increased to 21 (a 9.0-point annual increase, Figure 1D). Initiation of CT started with one half of the recommended $20 \mathrm{~g}$ /day dose in February 2011, and the patient was maintained on this and his pharmacologic treatments. The last available MMSE score was 18 in May 2012 (annual decline of 2.4 points, Figure 1D). The physician evaluated the patient as having moderate $\mathrm{AD}$ at the initiation of $\mathrm{CT}$ therapy, and after 15 months the physician rated the patient as stable. No adverse events related to CT were reported. The caregiver also assessed the patient as stable.

\section{Case 5}

The fifth patient had been genotyped for Apolipoprotein $E$ $(A P O E)$ and was e3/e4 (APOE4+). In June 2009, she presented with an MMSE of 26, and by July 2010, it had declined to 22 (annual change of -3.70 points). The patient was diagnosed with mild $\mathrm{AD}$, and both memantine $10 \mathrm{mg}$ twice daily and CT $20 \mathrm{~g} /$ day were initiated at this time. The patient was also treated with sertraline over the period from January to July 2012. The last available MMSE score for this patient was 27 and was recorded in July 2012 (annual change +2.60 points, Figure 1E). The treating physician rated the patient as having marked improvement over the course of 25 months. The caregiver rated the patient as having some improvement. No adverse events related to CT were reported.

\section{Case 6}

The sixth patient presented with an MMSE score of 29 in April 2009 and was treated with memantine $10 \mathrm{mg}$ twice daily. By October 2010, the MMSE score had declined to 23 (annual change -4.0 points) and the patient was started on CT $20 \mathrm{~g} /$ day. The patient continued on CT until May 2011 and his last MMSE score was 25 (annual change +3.4 points). He discontinued CT at that time due to mild gastrointestinal upset. The patient's most recent MMSE score in June 2012 (13 months after the end of CT) was 22 (Figure 1F). Over 6 months of CT, the treating physician's diagnosis of mild AD did not change and the patient was classified as stable. Caregiver assessment was not available.

\section{Case 7}

In August 2008, this patient presented with an MMSE of 24 and treatment was initiated with a $9.5 \mathrm{mg}$ rivastigmine patch and memantine $10 \mathrm{mg}$ twice daily. From August 2008 to July 2010, the patient's MMSE score declined to 22 and CT $20 \mathrm{~g}$ was added (annual change -1.0 points). This course of therapy is continuing, and the patient's last available MMSE score was 15 in July 2012 (annual change -3.5 points, Figure 1G). During the period over which CT has been administered, that patient has taken several other psychotropic medications, including venlafaxine, olanzapine, risperidone, alprazolam, divalproex sodium, and mirtazapine. The physician rated the patient as having moderate $\mathrm{AD}$ at the beginning of $\mathrm{CT}$ and severe $\mathrm{AD}$ at the end of this period. The change from baseline was moderate worsening. Caregiver assessments 
were available and indicated some improvement. No adverse events related to $\mathrm{CT}$ were reported.

\section{Case 8}

This patient was diagnosed with mild AD in January 2009 with an MMSE of 24. In November 2010, the patient's MMSE had declined to 22 (annual change -1.1 points, Figure $1 \mathrm{H})$. Treatment with donepezil $10 \mathrm{mg}$ once daily and memantine $10 \mathrm{mg}$ twice daily was initiated in January 2009, but stopped by the patient in March of that year. Donepezil was reinitiated in July 2009, titrated to $10 \mathrm{mg}$ once daily and increased to $23 \mathrm{mg}$ once daily in July 2011. Memantine $10 \mathrm{mg}$ twice daily was restarted in November 2011. The patient was also being treated with other psychotropic medications (citalopram, quetiapine, venlafaxine, and divalproex sodium). In November 2010, the patient had CT (one half of the recommended dose) added to therapy. His MMSE score at that time was 22. Therapy is ongoing and at the time of his most recent evaluation (December 2011), the MMSE score was 20 (annual change -1.8 points). The treating physician rated the patient as having moderate $\mathrm{AD}$ at the beginning and end of 13 months of CT and the patient's condition was classified as stable. Caregiver assessments were available and also indicated that the patient was stable. No adverse events related to $\mathrm{CT}$ were reported.

\section{Overall results for change in MMSE scores}

The mean (median) annual rates of decline prior to the initiation of CT were $-1.34(-2.95)$ and those after addition of CT to ongoing pharmacotherapy were $-0.64(-0.55)$. Comparison of rates of decline before and after addition of CT to treatment indicated no significant difference for the two periods ( $P=0.3735$, Mann-Whitney $U$-test).

\section{Discussion}

Results from the cases summarized suggest several distinct patterns of response to $\mathrm{CT}$ based on changes in MMSE scores. Several patients had stabilization (cases 3 and 6) or modest improvements (cases 2 and 5) after CT was added to therapy, while MMSE scores in others continued to decline (cases 1, 4, 7, and 8). Variability in response has been noted for $\mathrm{AD}$ treatments, and one important aspect of research regarding this disease is identification of factors that predict responsivity to therapy. ${ }^{13,14}$ Prior studies have demonstrated that $A P O E 4+$ patients are less responsive to CT than those who are $A P O E 4-{ }^{6}$ However, the single patient with a known APOE4 genotype in this study was APOE4+ and showed improvement in her MMSE score after initiation of CT. It has been shown that the $A P O E$ e 4 allele is a significant risk factor for $\mathrm{AD}$, but that this genotype is uncommon in the general population. ${ }^{15,16}$ Overall, the annual rate of MMSE decline decreased in four patients and increased in four patients after initiation of CT.

The mean (median) annual rates of decline prior to initiation of CT were $-1.34(-2.95)$ and those after addition of CT were $-0.64(-0.55)$ for the eight patients included in this report, and the difference between the rates of decline before and after addition of CT to therapy was not significant. Several studies with much larger patient samples have provided information regarding the decline in MMSE scores in patients who were and were not receiving AD-specific therapies. A review of studies published prior to 2005 indicated a variable rate of decline in MMSE scores of -0.6 to -4.4 points per year in patients with AD. ${ }^{17}$ The REAL.FR (REseau sur la maladie d'Alzheimer FRançais) cohort has been followed up twice annually with MMSE and the Alzheimer Disease Assessment Scale-cognitive subscale. A study published in 2011 that included 686 patients from the REAL.FR cohort ( $90 \%$ receiving AD-specific medication) demonstrated an annual decline in MMSE scores of -2.4 points per year. ${ }^{18}$ An earlier study of 938 patients with mild-to-moderate AD included in the Italian National Cronos Project, who were receiving donepezil, galantamine, or rivastigmine, indicated an average decline of -1 point over 36 weeks of follow-up (representing an annual decline of -1.4 points). ${ }^{19}$

While several of the cases included in this report suggest that addition of CT to pharmacotherapy may stabilize MMSE scores in patients with mild-to-moderate $A D$, it should be acknowledged that this measure has important limitations for assessment of cognitive function.

The rate of change for MMSE is known to be influenced by several factors including baseline score, age, duration of disease, education, and prior experience with the test; ${ }^{20-22}$ there were substantial differences in the rates of change for MMSE scores, both before and after addition of CT to therapy, in the eight patients included in this report.

The limitations of current pharmacotherapy have prompted interest in diet and dietary supplementation as part of the overall treatment regimen for patients with AD. Meta-analysis of results from six prospective studies published between 2006 and 2009 indicated that high adherence to the Mediterranean diet was associated with a significantly decreased risk for neurodegenerative diseases, including $\mathrm{AD},{ }^{23}$ but there is no evidence that it slows cognitive decline 
in patients with AD. Many dietary supplements have been evaluated in $\mathrm{AD}$, and there is both positive and negative evidence for omega-3 fatty acids, ${ }^{24,25}$ coenzyme $\mathrm{Q} /$ idebenone, ${ }^{26,27}$ and acetyl-L-carnitine. ${ }^{28,29}$

The CT preparation administered to patients in the cases described is composed of a glycerol backbone with three caprylic fatty acids (C8:0) esterified to the glycerol. Medium chain triglycerides have been used in specialty nutrition for over 40 years. They are commonly employed in patients who have difficulty metabolizing long chain triglycerides, eg, those with pancreatic insufficiency. In addition, medium chain triglycerides have been used in ketogenic diets for children with refractive epilepsy, due to their inherent ketogenic properties. ${ }^{30}$

\section{Conclusion}

Results from this case review study indicated that addition of CT to pharmacotherapy for AD was associated with stable disease or improvement for some patients. In particular, addition of CT seemed to have slowed the rate of decline, as measured by MMSE scores, compared with rates of decline reported in larger longitudinal studies. These findings warrant further investigation in a larger study.

\section{Disclosure}

This study was funded by Accera Inc. The sponsor was involved in the study design, data collection, analysis, and interpretation, and preparation and submission of the manuscript. The authors wish to acknowledge the medical writing and editorial support of Robert Rhoades provided by Accera Inc. SDM is a member of the Accera speakers' bureau. JG is a member of the Accera speakers' bureau and scientific advisory board. The authors report no other conflicts of interest in this work.

\section{References}

1. Alzheimer's Association. 2012 Alzheimer's Disease Facts and Figures. Chicago, IL: Alzheimer's Association; 2012.

2. Salomone S, Caraci F, Leggio GM, Fedotova J, Drago F. New pharmacological strategies for treatment of Alzheimer's disease: focus on disease modifying drugs. Br J Clin Pharmacol. 2012;73(4):504-517.

3. US Food and Drug Administration. Guidance for Industry: Frequently Asked Questions About Medical Foods. 2007. Available from: http:// www.fda.gov/food/guidanceregulation/guidancedocumentsregulatoryinformation/medicalfoods/ucm054048.htm. Accessed March 16, 2013.

4. Scheltens P, Kamphuis PJ, Verhey FR, et al. Efficacy of a medical food in mild Alzheimer's disease: a randomized, controlled trial. Alzheimers Dement. 2010;6(1):1-10.

5. Chan A, Paskavitz J, Remington R, Rasmussen S, Shea TB. Efficacy of a vitamin/nutriceutical formulation for early-stage Alzheimer's disease: a 1-year, open-label pilot study with an 16-month caregiver extension. Am J Alzheimers Dis Other Demen. 2008;23(6):571-585.
6. Henderson ST, Vogel JL, Barr LJ, Garvin F, Jones JJ, Costantini LC. Study of the ketogenic agent AC-1202 in mild to moderate Alzheimer's disease: a randomized, double-blind, placebo-controlled, multicenter trial. Nutr Metab. 2009;6(1):31.

7. Reger MA, Henderson ST, Hale C, et al. Effects of beta-hydroxybutyrate on cognition in memory-impaired adults. Neurobiol Aging. 2004;25(3): 311-314.

8. Costantini LC, Barr LJ, Vogel JL, Henderson ST. Hypometabolism as a therapeutic target in Alzheimer's disease. BMC Neurosci. 2008; 9 Suppl 2:S16.

9. Cunnane S, Nugent S, Roy M, et al. Brain fuel metabolism, aging, and Alzheimer's disease. Nutrition. 2011;27(1):3-20.

10. Henderson ST. Ketone bodies as a therapeutic for Alzheimer's disease. Neurotherapeutics. 2008;5(3):470-480.

11. Babayan VK. Medium chain triglycerides and structured lipids. Lipids. 1987;22(6):417-420.

12. Maynard SD, Gelblum J. Retrospective cohort study of the efficacy of caprylic triglyceride in patients with mild-to-moderate Alzheimer's disease. Neuropsychiatr Dis Treat. 2013;9:1619-1627.

13. Kanetaka H, Hanyu H, Hirao K, et al. Prediction of response to donepezil in Alzheimer's disease: combined MRI analysis of the substantia innominata and SPECT measurement of cerebral perfusion. Nucl Med Commun. 2008;29(6):568-573.

14. Cacabelos R. Molecular pathology and pharmacogenomics in Alzheimer's disease: polygenic-related effects of multifactorial treatments on cognition, anxiety and depression. Methods Find Exp Clin Pharmacol. 2007;29 Suppl A:1-91.

15. Hsiung GY, Sadovnick AD, Feldman H. Apolipoprotein E epsilon4 genotype as a risk factor for cognitive decline and dementia: data from the Canadian Study of Health and Aging. CMAJ. 2004;171(8):863-867.

16. Myers RH, Schaefer EJ, Wilson PW, et al. Apolipoprotein E epsilon4 association with dementia in a population-based study: The Framingham study. Neurology. 1996;46(3):673-677.

17. Behl P, Stefurak TL, Black SE. Progress in clinical neurosciences: cognitive markers of progression in Alzheimer's disease. Can J Neurol Sci. 2005;32(2):140-151.

18. Gillette-Guyonnet S, Andrieu S, Nourhashemi F, et al. Long-term progression of Alzheimer's disease in patients under antidementia drugs. Alzheimers Dement. 2011;7(6):579-592.

19. Santoro A, Siviero P, Minicuci N, et al. Effects of donepezil, galantamine and rivastigmine in 938 Italian patients with Alzheimer's disease: a prospective, observational study. CNS Drugs. 2010;24(2):163-176.

20. Tombaugh TN, McIntyre NJ. The Mini-Mental State Examination: a comprehensive review. J Am Geriatr Soc. 1992;40(9):922-935.

21. Lancu I, Olmer A. [The Mini-Mental State Examination - an up-to-date review.] Harefuah. 2006;145(9):687-690. Hebrew.

22. Unger JM, van Belle G, Heyman A. Cross-sectional versus longitudinal estimates of cognitive change in nondemented older people: a CERAD study. Consortium to Establish a Registry for Alzheimer's Disease. J Am Geriatr Soc. 1999;47(5):559-563.

23. Sofi F, Abbate R, Gensini GF, Casini A. Accruing evidence on benefits of adherence to the Mediterranean diet on health: an updated systematic review and meta-analysis. Am J Clin Nutr. 2010;92(5): 1189-1196.

24. Issa AM, Mojica WA, Morton SC, et al. The efficacy of omega-3 fatty acids on cognitive function in aging and dementia: a systematic review. Dement Geriatr Cogn Disord. 2006;21(2):88-96.

25. Quinn JF, Raman R, Thomas RG, et al. Docosahexaenoic acid supplementation and cognitive decline in Alzheimer disease: a randomized trial. JAMA. 2010;304(17):1903-1911.

26. Weyer G, Babej-Dölle RM, Hadler D, Hofmann S, Herrmann WM. A controlled study of 2 doses of idebenone in the treatment of Alzheimer's disease. Neuropsychobiology. 1997;36(2):73-82.

27. Thal LJ, Grundman M, Berg J, et al. Idebenone treatment fails to slow cognitive decline in Alzheimer's disease. Neurology. 2003;6(11): 1498-1502. 
28. Montgomery SA, Thal LJ, Amrein R. Meta-analysis of double blind randomized controlled clinical trials of acetyl-L-carnitine versus placebo in the treatment of mild cognitive impairment and mild Alzheimer's disease. Int Clin Psychopharmacol. 2003;18(2):61-71.

29. Hudson S, Tabet N. Acetyl-L-carnitine for dementia. Cochrane Database Syst Rev. 2003;2:CD003158.
30. Liu YM, Wang HS. Medium-chain triglyceride ketogenic diet, an effective treatment for drug-resistant epilepsy and a comparison with other ketogenic diets. Biomed J. 2013;36(1):9-15.

Neuropsychiatric Disease and Treatment

\section{Publish your work in this journal}

Neuropsychiatric Disease and Treatment is an international, peerreviewed journal of clinical therapeutics and pharmacology focusing on concise rapid reporting of clinical or pre-clinical studies on a range of neuropsychiatric and neurological disorders. This journa is indexed on PubMed Central, the 'PsycINFO' database and CAS
The manuscript management system is completely online and includes a very quick and fair peer-review system, which is all easy to use. Visit http://www.dovepress.com/testimonials.php to read real quotes from published authors.

Submit your manuscript here: http://www.dovepress.com/neuropsychiatric-disease-and-treatment-journal 